\title{
The Space of Continuous Periodic Functions Is a Set of First Category in $A P(X)$
}

\author{
Zhe-Ming Zheng, ${ }^{1}$ Hui-Sheng Ding, ${ }^{1}$ and Gaston M. N'Guérékata ${ }^{2}$ \\ ${ }^{1}$ College of Mathematics and Information Science, Jiangxi Normal University, Nanchang, Jiangxi 330022, China \\ ${ }^{2}$ Department of Mathematics, Morgan State University, 1700 E. Cold Spring Lane, Baltimore, MD 21251, USA
}

Correspondence should be addressed to Hui-Sheng Ding; dinghs@mail.ustc.edu.cn

Received 17 January 2013; Accepted 12 February 2013

Academic Editor: Józef Banaś

Copyright (c) 2013 Zhe-Ming Zheng et al. This is an open access article distributed under the Creative Commons Attribution License, which permits unrestricted use, distribution, and reproduction in any medium, provided the original work is properly cited.

We prove that the space of continuous periodic functions is a set of first category in the space of almost periodic functions, and we also show that the space of almost periodic functions is a set of first category in the space of almost automorphic functions.

\section{Introduction}

Since the last century, the study on almost periodic type functions and their applications to evolution equations has been of great interest for many mathematicians. There is a large literature on this topic. Several books are especially devoted to almost periodic type functions and their applications to differential equations and dynamical systems. For example, let us indicate the books of Amerio and Prouse [1], Bezandry and Diagana [2], Bohr [3], Corduneanu [4], Diagana [5], Fink [6], Levitan and Zhikov [7], N'Guérékata [8, 9], Pankov [10], Shen and Yi [11], Zaidman [12], and Zhang [13].

Although almost periodic functions have a very wide range of applications now, it seems that giving an example of almost periodic (not periodic) functions is more difficult than giving an example of periodic functions. Also, there is a similar problem for almost automorphic functions. In this paper, we aim to compare the "amount" of almost periodic functions (not periodic) with the "amount" of continuous periodic functions, and we also discuss the related problems for almost automorphic functions.

\section{Main Results}

Throughout the rest of this paper, we denote by $\mathbb{R}$ the set of real numbers, by $X$ a Banach space, and by $C(\mathbb{R}, X)$ the set of all continuous functions $f: \mathbb{R} \rightarrow X$.
Definition 1 (see [4]). A function $f \in C(\mathbb{R}, X)$ is called almost periodic if, for every $\varepsilon>0$, there exists $l(\varepsilon)>0$ such that every interval of length $l(\varepsilon)$ contains a number $\tau$ with the property that

$$
\sup _{t \in \mathbb{R}}\|f(t+\tau)-f(t)\|<\varepsilon .
$$

We denote the collection of all such functions by $A P(X)$.

Recall that $A P(X)$ is a Banach space under the supremum norm.

Definition 2. A function $f \in C(\mathbb{R}, X)$ is called periodic if there exists $l>0$ such that

$$
f(t+l)=f(t), \quad \forall t \in \mathbb{R} .
$$

Here, $l$ is called a period of $f$. We denote the collection of all such functions by $P(X)$. For $f \in P(X)$, we call $l_{0}$ the fundamental period if $l_{0}$ is the smallest period of $f$.

Remark 3. Similar to the proof in [4, page 1], it is not difficult to show that if $f \in P(X)$ is not constant, and then $f$ has the fundamental period. 
Definition 4 (see [8]). A function $f \in C(\mathbb{R}, X)$ is called almost automorphic if, for every real sequence $\left(s_{m}\right)$, there exists a subsequence $\left(s_{n}\right)$ such that

$$
g(t)=\lim _{n \rightarrow \infty} f\left(t+s_{n}\right)
$$

is well defined for each $t \in \mathbb{R}$ and

$$
\lim _{n \rightarrow \infty} g\left(t-s_{n}\right)=f(t)
$$

for each $t \in \mathbb{R}$. Denote by $A A(X)$ the set of all such functions.

Recall that there exists an almost automorphic function which is not almost periodic, for instance, the following function:

$$
f(t)=\sin \frac{1}{2+\cos t+\cos \sqrt{2} t}, \quad t \in \mathbb{R} .
$$

Before the proof of our main results, we need to recall the notion about the first category.

Definition 5 (see [14]). Let $S$ be a topological space. A set $E \subset S$ is said to be nowhere dense if its closure has an empty interior. The sets of the first category in $S$ are those that are countable unions of nowhere dense sets. Any subset of $S$ that is not of the first category is said to be of the second category in $S$.

Theorem 6. $P(X)$ is a set of first category in $A P(X)$.

Proof. For $n=1,2, \ldots$, we denote

$$
\begin{gathered}
P_{n}=\{f \in C(\mathbb{R}, X): \text { there exists } l \in[n, n+1] \\
\text { such that } f(t+l)=f(t) \quad \forall t \in \mathbb{R}\} .
\end{gathered}
$$

Then, it is easy to see that

$$
P(X)=\bigcup_{n=1}^{\infty} P_{n}
$$

We divide the remaining proof into two steps.

Step 1. Every $P_{n}$ is a closed subset of $A P(X)$.

Let $f \in A P(X) \backslash P_{n}$. Then, for every $l \in[n, n+1]$, there exists $t_{l} \in \mathbb{R}$ such that $f\left(t_{l}+l\right) \neq f\left(t_{l}\right)$. Denote

$$
\varepsilon_{l}:=\frac{1}{4}\left\|f\left(t_{l}+l\right)-f\left(t_{l}\right)\right\|>0, \quad l \in[n, n+1] .
$$

In addition, due to the continuity of $f$, for every $l \in[n, n+1]$, there exists $\delta_{l}>0$ such that

$$
\left\|f\left(t_{l}+s\right)-f\left(t_{l}\right)\right\| \geq 3 \varepsilon_{l}, \quad \forall s \in\left(l-\delta_{l}, l+\delta_{l}\right) .
$$

Obviously, we have

$$
[n, n+1] \subset \bigcup_{l \in[n, n+1]}\left(l-\delta_{l}, l+\delta_{l}\right) .
$$

Then, by the Heine-Borel theorem, there exists $l_{1}, \ldots, l_{k} \in$ $[n, n+1]$ such that

$$
[n, n+1] \subset \bigcup_{i=1}^{k}\left(l_{i}-\delta_{l_{i}}, l_{i}+\delta_{l_{i}}\right),
$$

where $k$ is a fixed positive integer. Letting $\varepsilon=\min _{1 \leq i \leq k}\left\{\varepsilon_{l_{i}}\right\}$, and

$$
N(f, \varepsilon):=\left\{g \in A P(X):\|g-f\|_{A P(X)}<\varepsilon\right\},
$$

for every $g \in N(f, \varepsilon)$, we claim that $g \notin P_{n}$. In fact, for every $l \in[n, n+1]$, there exists $i \in\{1, \ldots, k\}$ such that

$$
l \in\left(l_{i}-\delta_{l_{i}}, l_{i}+\delta_{l_{i}}\right) \text {. }
$$

Then, by (9), we have

$$
\left\|f\left(t_{l_{i}}+l\right)-f\left(t_{l_{i}}\right)\right\| \geq 3 \varepsilon_{l_{i}} \geq 3 \varepsilon,
$$

which yields that

$$
\begin{aligned}
& \left\|g\left(t_{l_{i}}+l\right)-g\left(t_{l_{i}}\right)\right\| \\
& \quad \geq\left\|f\left(t_{l_{i}}+l\right)-f\left(t_{l_{i}}\right)\right\|-\left\|f\left(t_{l_{i}}+l\right)-g\left(t_{l_{i}}+l\right)\right\| \\
& \quad-\left\|f\left(t_{l_{i}}\right)-g\left(t_{l_{i}}\right)\right\| \\
& \quad \geq 3 \varepsilon-\varepsilon-\varepsilon=\varepsilon>0,
\end{aligned}
$$

where $\|g-f\|_{A P(\mathbb{R})}<\varepsilon$ was used. So, we know that $N(f, \varepsilon) \subset$ $A P(X) \backslash P_{n}$, which means that $P_{n}$ is a closed subset of $A P(X)$.

Step 2. Every $P_{n}$ has an empty interior.

It suffices to prove that, for every $f \in P_{n}$ and $\delta>0$, $N(f, \delta) \bigcap\left(A P(X) \backslash P_{n}\right) \neq \emptyset$. Now let $f \in P_{n}$ and $\delta>0$. In the following, we discuss two cases.

Case I. $f$ is constant.

We denote

$$
f_{\delta}(t)=\frac{\cos t+\cos (\sqrt{2} t)}{3} \cdot \delta \cdot x_{0}+f(t), \quad t \in \mathbb{R},
$$

where $x_{0} \in X$ is some constant with $\left\|x_{0}\right\|=1$. Then $f_{\delta} \in$ $N(f, \delta)$ and $f_{\delta} \notin P_{n}$ since $f_{\delta}$ is not periodic.

Case II. $f$ is not constant.

By Remark 3, $f$ has a fundamental period $l_{0}$. We denote

$$
f_{\delta}(t)=f(t)+f\left(\frac{t}{\pi}\right) \cdot \frac{\delta}{M_{f}}, \quad t \in \mathbb{R},
$$

where $M_{f}=\sup _{t \in \mathbb{R}}\|f(t)\|$. Obviously, $f_{\delta} \in N(f, \delta)$. Also, we claim that $f_{\delta} \notin P_{n}$. In fact, if this is not true, then there exists $T \in[n, n+1]$ such that

$$
f_{\delta}(t+T)=f_{\delta}(t), \quad t \in \mathbb{R}
$$

that is,

$$
f(t+T)+f\left(\frac{t+T}{\pi}\right) \cdot \frac{\delta}{M_{f}}=f(t)+f\left(\frac{t}{\pi}\right) \cdot \frac{\delta}{M_{f}}, \quad t \in \mathbb{R} .
$$

Let

$$
\begin{gathered}
F_{1}(t)=f(t+T)-f(t), \\
F_{2}(t)=\frac{\delta}{M_{f}}\left[f\left(\frac{t}{\pi}\right)-f\left(\frac{t+T}{\pi}\right)\right], \quad t \in \mathbb{R} .
\end{gathered}
$$


Then $F_{1}(t) \equiv F_{2}(t)$. If $F_{1}(t) \equiv F_{2}(t) \equiv C$, where $C$ is a fixed constant, then

$$
f(t+T)=f(t)+C, \quad t \in \mathbb{R},
$$

which yields

$$
C=\frac{f(k T)-f(0)}{k} \longrightarrow 0, \quad k \longrightarrow \infty
$$

since $f$ is bounded. Thus, we have

$$
f(t+T)=f(t), \quad f\left(\frac{t}{\pi}\right)=f\left(\frac{t+T}{\pi}\right), \quad t \in \mathbb{R} .
$$

Noting that $l_{0}$ is the fundamental period of $f$ and $\pi l_{0}$ is the fundamental period of $f(\cdot / \pi)$, there exist two positive integers $p$ and $q$ such that

$$
p l_{0}=T=q \pi l_{0}
$$

that is, $\pi=p / q$, which is a contradiction. If $F_{1}=F_{2}$ is not constant, then, by Remark 3, we can assume that $T_{0}$ is the fundamental period of $F_{1}$ and $F_{2}$. Noting that $l_{0}$ is a period of $F_{1}$ and $\pi l_{0}$ is a period of $F_{2}$, similar to the above proof, we can also show that $\pi$ is a rational number, which is a contradiction.

In conclusion, $P(X)$ is countable unions of closed subsets with empty interior. So $P(X)$ is a set of first category.

Remark 7. Since $A P(X)$ is a set of second category, it follows from Theorem 6 that $A P(X) \backslash P(X)$ is a set of second category, which means that, to some extent, the "amount" of almost periodic functions (not periodic) is far more than the "amount" of continuous periodic functions.

Theorem 8. $A P(X)$ is a set of first category in $A A(X)$.

Proof. Firstly, $A P(X)$ is a closed subset of $A A(X)$. Secondly, $A P(X)$ has an empty interior in $A A(X)$. In fact, letting

$$
\varphi(t)=\sin \frac{1}{2+\cos t+\cos (\sqrt{2} t)}, \quad t \in \mathbb{R},
$$

for every $f \in A P(X)$ and $\delta>0$, we have $f_{\delta} \notin A P(X)$ and $f_{\delta} \in N(f, \delta)$, where

$$
f_{\delta}(t)=f(t)+\frac{\delta x_{0}}{M_{\varphi}} \varphi(t), \quad t \in \mathbb{R}, \quad M_{\varphi}=\sup _{t \in \mathbb{R}}\|\varphi(t)\|,
$$

and $x_{0} \in X$ is some constant with $\left\|x_{0}\right\|=1$. This completes the proof.

Remark 9. By Theorem $8, A A(X) \backslash A P(X)$ is a set of second category in $A A(X)$, which means that, to some extent, the "amount" of almost automorphic functions (not almost periodic) is far more than the "amount" of almost periodic functions.

\section{Acknowledgments}

H.-S. Ding acknowledges support from the NSF of China (11101192), the Chinese Ministry of Education (211090), the NSF of Jiangxi Province (20114BAB211002), and the Jiangxi Provincial Education Department (GJJ12173).

\section{References}

[1] L. Amerio and G. Prouse, Almost-Periodic Functions and Functional Equations, The University Series in Higher Mathematics, Van Nostrand Reinhold, New York, NY, USA, 1971.

[2] P. H. Bezandry and T. Diagana, Almost Periodic Stochastic Processes, Springer, New York, NY, USA, 2011.

[3] H. Bohr, Almost Periodic Functions, Chelsea Publishing, New York, NY, USA, 1947.

[4] C. Corduneanu, Almost Periodic Functions, Chelsea Publishing, New York, NY, USA, 2nd edition, 1989.

[5] T. Diagana, Pseudo Almost Periodic Functions in Banach Spaces, Nova Science, New York, BY, USA, 2007.

[6] A. M. Fink, Almost Periodic Differential Equations, vol. 377 of Lecture Notes in Mathematics, Springer, Berlin, Germany, 1974.

[7] B. M. Levitan and V. V. Zhikov, Almost Periodic Functions and Differential Equations, Cambridge University Press, Cambridge, UK, 1982.

[8] G. M. N'Guérékata, Almost Automorphic and Almost Periodic Functions in Abstract Spaces, Kluwer Academic, New York, NY, USA, 2001.

[9] G. M. N'Guérékata, Topics in Almost Automorphy, Springer, New York, NY, USA, 2005

[10] A. A. Pankov, Bounded and Almost Periodic Solutions of Nonlinear Operator Differential Equations, vol. 55 of Mathematics and its Applications (Soviet Series), Kluwer Academic, Dordrecht, The Netherlands, 1990.

[11] W. X. Shen and Y. F. Yi, Almost Automorphic and Almost Periodic Dynamics in Skew-Product Semiflows, no. 647 of Memoirs of the American Mathematical Society, 1998.

[12] S. Zaidman, Almost-Periodic Functions in Abstract Spaces, vol. 126 of Research Notes in Mathematics, Pitman, Boston, Mass, USA, 1985.

[13] C. Zhang, Almost Periodic Type Functions and Ergodicity, Kluwer Academic, Dordrecht, The Netherlands, 2003.

[14] W. Rudin, Functional Analysis, International Series in Pure and Applied Mathematics, McGraw-Hill, New York, NY, USA, 2nd edition, 1991. 


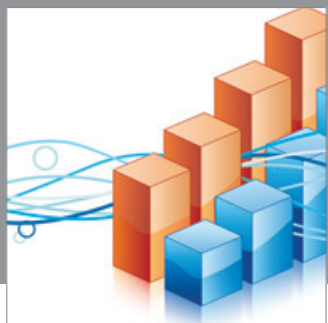

Advances in

Operations Research

mansans

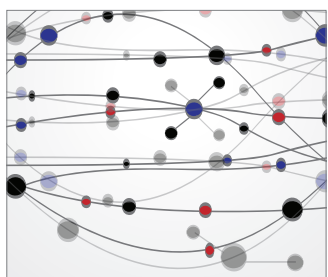

The Scientific World Journal
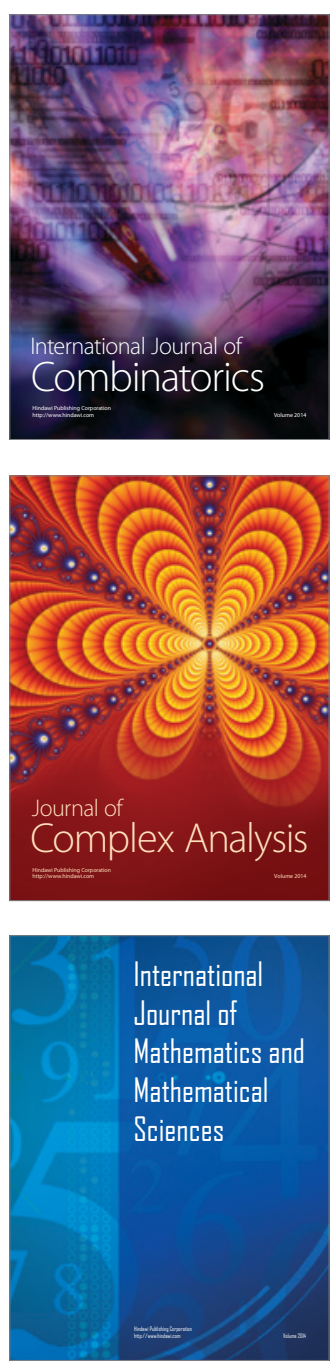
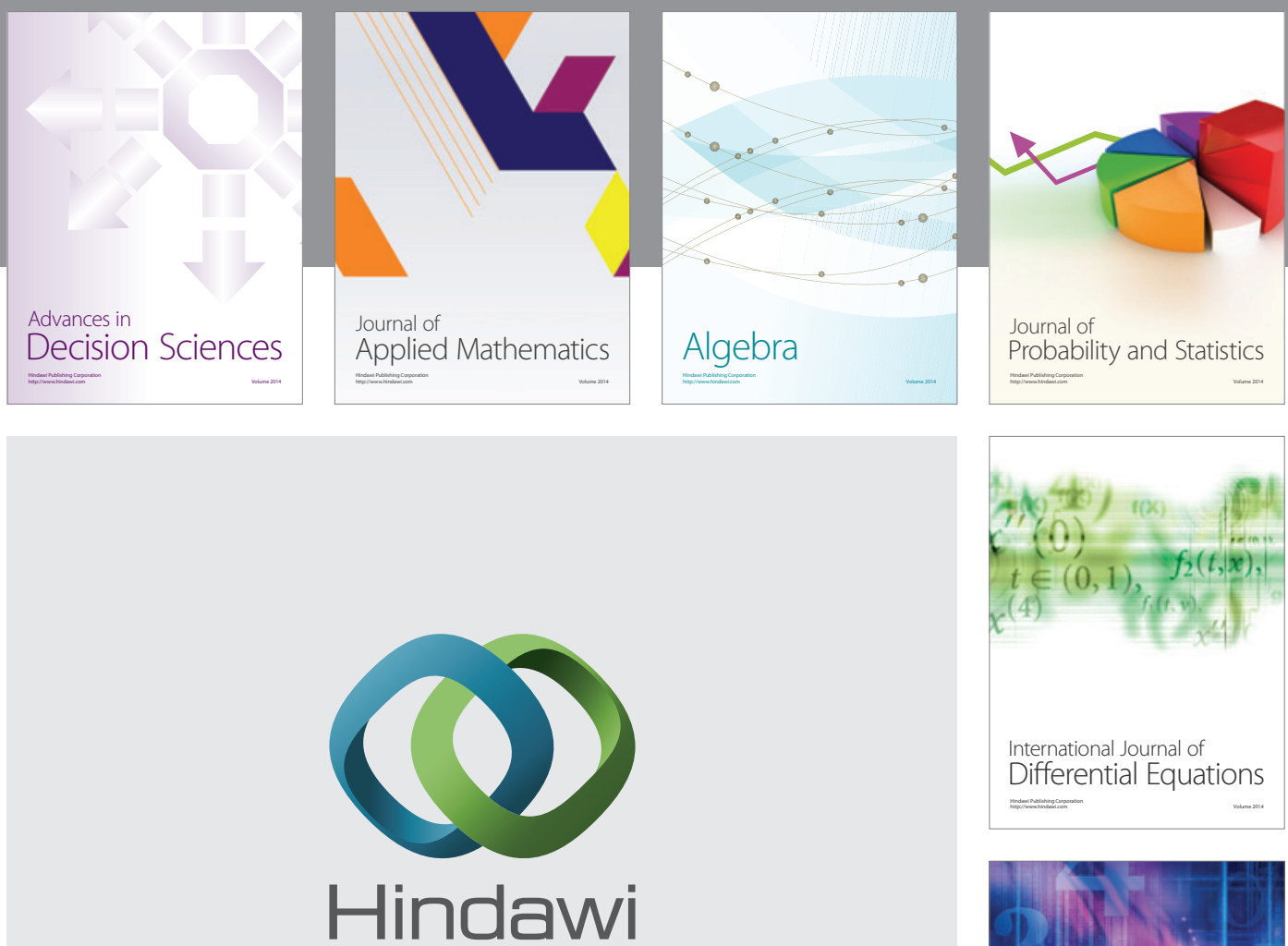

Submit your manuscripts at http://www.hindawi.com
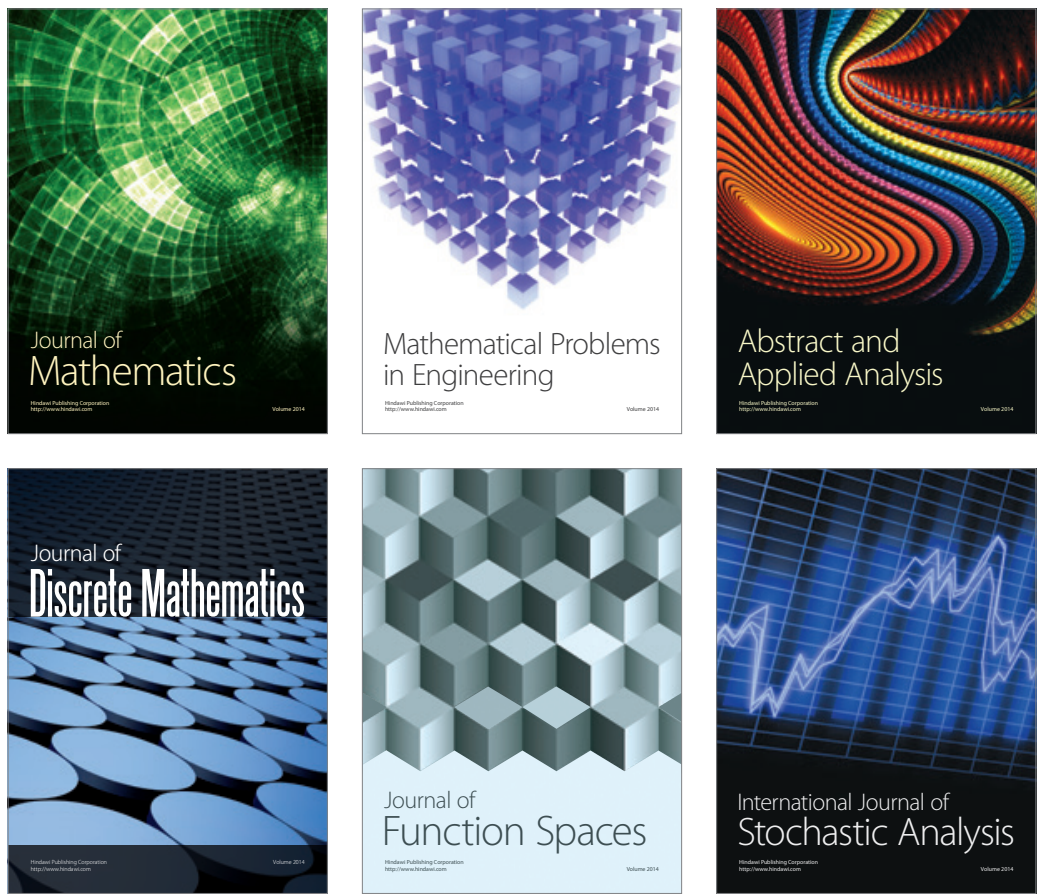

Journal of

Function Spaces

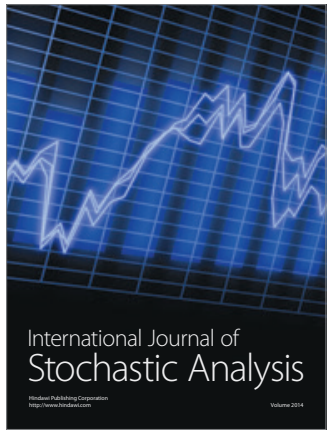

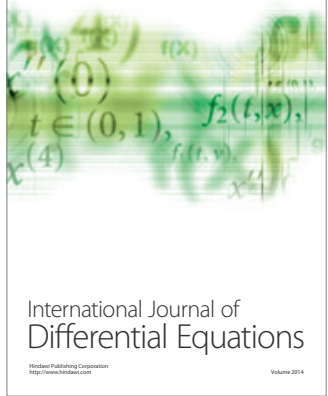
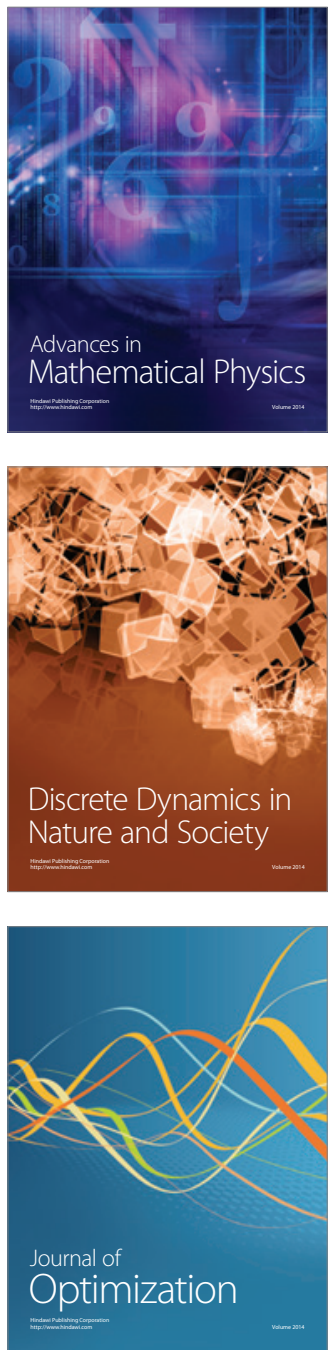